\title{
Venous Scenario in India
}

\author{
Ajay K. Khanna ${ }^{1}$ (D) Sandeep Kumar ${ }^{2}$
}

Received: 12 May 2021 / Accepted: 14 May 2021 / Published online: 12 June 2021

(C) The Author(s) 2021

\begin{abstract}
Varicose veins, venous thrombosis, and other venous diseases are equally prevalent in India as anywhere else in the world. Scientific and epidemiological studies of venous diseases in India are being made but at a slow pace. It requires to be enhanced. To boost such efforts, the Vascular Society of India came into existence in 1995 but little progress has been made in advancing the speciality and care for venous diseases in India. Though Vascular Society of India also deals with the venous component but to be more specific and emphatic the Venous Association of India came into existence. In April 2007, seven surgeons met to create the organization's bylaws and registered the Venous Association of India in Chennai, Tamil Nadu, India, with the aim to promote the research and study of venous disease. These initiatives have recruited many surgeons looking into new vistas of sub or super specialization to join in the folds of Venous Association of India. These efforts have surely shown some results. As, several Indian authors have published books on venous diseases.
\end{abstract}

Keywords Vein · Epidemiology $\cdot$ Deep vein thrombosis $\cdot$ Chronic venous insufficiency

\section{Introduction}

Varicose veins, venous thrombosis, and other venous diseases are equally prevalent in India as anywhere else in the world. Scientific and epidemiological studies of venous diseases in India are being made but at a slow pace. It requires to be enhanced. To boost such efforts, the Vascular Society of India came into existence in 1995 but little progress has been made in advancing the speciality and care for venous diseases in India. The aim of the Vascular Society of India with its head office at Chennai are as follows: to encourage and advance the study and practice of the science and art of vascular surgery, to promote interaction among vascular surgeons and allied specialists in India and abroad, to encourage scientific research and experimental work on vascular disease, to organize scientific programs such as conferences and workshops, to be a resource for continuing medical education programs, audiovisual materials, etc. related to vascular surgery, to establish and

Ajay K. Khanna

akhannabhu@gmail.com

1 Department of General Surgery Institute of Medical Sciences, Banaras Hindu University, 221005 Varanasi, India

2 Department of General Surgery, King Georges Medical University, Lucknow, India maintain reference libraries of books, magazines, newspapers, audiovisual materials, etc. related to vascular surgery, and to publish a journal devoted to vascular surgery and allied subjects. The Indian Journal of Vascular and Endovascular Surgery (IJVES) was founded in 2014 published quarterly as the official publication of the Vascular Society of India. This journal looks after the various vascular articles and also the work related to venous diseases. Though Vascular Society of India also deals with the venous component but to be more specific and emphatic, the Venous Association of India came into existence.

\section{Venous Association of India (VAI)}

In 2004, several surgeons began thinking about forming an association of care providers and other healthcare professionals who had a special interest in venous diseases in India. In line with American Venous Forum, the European Venous Forum, and other Phlebological Societies, a dedicated VAI was proposed. At that time, discussions about venous disease in India were primarily confined to a single session or two at the annual meetings of the Vascular Society of India. The first program that focused on venous disease in India was the Continuing Surgical Education Program in Vascular Surgery and Venous Diseases that was held at Christian 
Medical College in Vellore, India, in 2005. In April 2007, seven surgeons met to create the organization's bylaws and registered the Venous Association of India in Chennai, Tamil $\mathrm{Nadu}$, India, with the following aims: to promote the research and study of venous disease, to help facilitate the active continuing education of its members, to improve understanding about the prevention, investigation, and treatment of venous disease, to encourage the development and dissemination of information about venous disease, to promote collaboration between venous, phlebological, and vascular societies, health agencies, and health experts at national and international levels, to award scholarships and stipends for research and training in the field of venous disease, and to cooperate with any other organization whose objectives are similar to those of the VAI. The first Executive Committee was initially comprised of seven members. This association holds its annual meeting every year usually in the month of February and March and this is a 3-day-program where only venous problems are discussed. The first conference was held at Chennai. Approximately, 20-30 overseas visitors from all over the world attend this meeting. This year in 2021, it was held virtually because of COVID pandemic. These initiatives have recruited many surgeons looking into new vistas of sub or super specialization to join in the folds of Venous Association of India. These efforts have surely shown some results. As, several Indian authors have published books on venous diseases [1-6].

\section{Varicose Veins}

The importance of identifying and treating the venous disease is getting better with increasing use of ultrasound and color Doppler duplex assessment, endovenous therapies, adequate anticoagulation, compression therapies, thrombo-prophylactic measures, and venous ulcer care. These worldwide developments in the advanced countries are now also being adopted and adapted in Asian countries and India. However, more epidemiological studies are needed to assess the incidence and natural course of the venous disease in different populations and effect of various treatments on the natural course of venous diseases. Even the study of anatomical aspects of the venous system is necessary to be studied in more details [7]. Proper treatment and research for venous diseases has been a long unmet need in India and many other countries. It is relatively an easier, less expensive, and not requiring major infrastructure sub-specialty which can be taken up by general surgeons practicing in smaller districts and rural areas also.

Varicose veins are part of the spectrum of chronic venous disease and include spider telangiectasias, reticular veins, and true varicosities. Approximately, $23 \%$ of US adults have varicose veins. If spider telangiectasias and reticular veins are also considered, the prevalence increases to $80 \%$ men and
$85 \%$ women. Generally, more common in women and older adults, varicose veins affect 22 million women and 11 million men between the ages of 40 and 80 years. Two million men and women will develop symptoms and signs of chronic venous insufficiency, including venous ulceration $[8,9]$.

An epidemiological survey carried out way back in 1972 on Indian railroad workers determined the prevalence of varicose veins to be $25 \%$ in southern and $6.8 \%$ in northern India [10].

A study from Mangalore, 170 varicose vein cases admitted in tertiary care hospitals between May 2011 and April 2014. Majority of cases 53 (31.2\%) were of the age group 41 to 50 years. Majority were males $127(74.7 \%)$ and majority were unskilled workers 101 (59.4\%). Superficial veins were involved in 123 (72.4\%) cases. Perforator followed by great saphenous vein was most frequently involved. Veins on the left side were more involved than on the right. Common symptoms at the time of presentation was ulceration 98 (57.6\%) followed by pain in the legs $96(56.5 \%)$. Ulceration was seen significantly more among women $(\mathrm{p}<0.027)$ and house wives $(\mathrm{p}<0.004)$. Complications like eczema 46 (27.1\%), non-healing ulcers 21 (12.3\%), and deep vein thrombosis $10(5.9 \%)$ were reported in these cases. Eczema was present significantly more among elderly patients aged above 60 years $(\mathrm{p}<0.019)$. Risk factors like prolonged standing was observed in $86(50.6 \%)$ cases. This history was significantly seen among men $(\mathrm{p}<0.001)$ and those involved in unskilled occupations $(\mathrm{p}<0.001)$. Recurrence of varicose vein was reported in $23(13.5 \%)$ cases. It was associated with younger age group 21 to 30 years $(\mathrm{p}<0.021)$. Doppler ultrasound was the most common 120 (70.6\%) investigation done. Micronized purified flavonoid fraction was used in management in 15 (8.8\%) cases. Medical management methods like limb elevation 50 (29.4\%) and compression stocking 36 (21.2\%) was advised to patients. Saphenous vein stripping was the most common surgical procedure 40 (23.5\%) performed. The high risk groups identified in this study need to be made aware of risk of developing varicose veins. Use of compression stocking at work place added with newer procedure in management could help in betterment in their quality of life [11].

In the western scenario, it has been found that incidence of varicose veins is higher in women [12]. But on the contrast in India, it is seen more in men. A study from D Y Patil Hospital, Pune, found $70 \%$ of patients were men, and only $30 \%$ were women [13]. Similar findings were from Rohtak where Vashist et al. showed $64 \%$ males and $36 \%$ women [14].

Although several reasons have been hypothesized for the varicose veins but bacterial infective pathology for varicose veins was not thought off up until a study done at Banaras Hindu University on 42 truncal veins and 113 non truncal veins. In 42 truncal veins, 9 specimens were sterile and 17 (40.4\%) specimens showed growth of Micrococci sp.; 6 
(14.3\%) Pseudomonas sp.; 4 (9.5\%) Klebsiella sp.; and 2 (4.7\%) Proteus sp. In 113 non truncal veins, 31 specimens were sterile while 31 (27.43\%) showed Micrococci spp., 14 (12.4\%) Pseudomonas spp., 12 (10.6\%) Staphylococcus aureus, $6(5.31 \%)$ Klebsiella spp. To confirm the findings of culture, DNA PCR was done with specific for named bacteria. In 42 trunkal veins, 10 (23.8\%) Pseudomonas, 6 (14.3\%) Staphylococcus, 5 (11.9\%) Klebsiella, 2 (4.8\%) Proteus, and $1(2.4 \%)$ Citrobacter were detected. In non-trunkal veins in 113 specimens, 25 (22.1\%) Pseudomonas, 19 (16.8\%) Staphylococcus, 7 (6.2\%) Klebsiella, 2 (1.8\%) Proteus, and $1(0.9 \%)$ Citrobacter were detected. Although bacterial etiology of varicose veins has not been studied well enough till date, in consideration of the results of above study, bacterial etiology of varicose veins is highly probable [15]. The same group also looked into the various inflammatory markers. Blood withdrawn from the site of varicose vein appears to have significantly increased concentration of interleukin-6, fibrinogen, and hemoglobin when compared to same patient's antecubital venous blood sample supporting the hypothesis of greater inflammation in tissues drained by varicose veins [16].

In India, still many centers offer open surgical treatment but in the metro cities and big cities, many centers are carrying out minimal invasive techniques for ablation of varicose veins as endovenous ablation by sclerotherapy and laser, endovenous radiofrequency ablation [17]. Few centers have started the endovenous steam sclerosis and microwave ablation. As these techniques require tumescent anesthesia so newer techniques as glue injection and mechanochemical ablation (MOCA) have also been used.

\section{Chronic Venous Insufficiency (CVD)}

CVD is one of the most common vascular disorders observed across the world and can severely impact life style (QOL), reduce patients' working hours, and impose a socioeconomic burden. Nonetheless, despite the capability of CVD to cause grave consequences, it remains one of the prominently underestimated and neglected diseases. Knowing CVD's potent disruptive nature, it is paramount to give it serious consideration and to define proper measures to prevent and/or manage it.

High prevalence, cost of investigation and treatment, and loss of working days due to CVD have a significant socioeconomic impact, and the problem is compounded because chronic venous insufficiency (CVI) is progressive and has a propensity to recur. The economic burden of CVD treatment is attributed to direct costs, such as medical and nursing costs and costs of investigation and treatment, and indirect costs, such as those related to loss of working days. Several studies carried out in different countries have shown that CVD incurs a significant economic burden. However, studies evaluating the incidence of CVD in India are limited. An epidemiological survey carried out way back in 1972 on Indian railroad workers determined the prevalence of varicose veins to be $25 \%$ in southern and $6.8 \%$ in northern India [10].

As the CVD is quite prevalent so there should be definite criteria which can define the role of intervention. The type of afflicted veins and the size of theveins can define that which patients may require intervention. Demographic characteristics and risk factors were recorded from 6350 patients. Patients with reflux in smaller veins (vein size $<4 \mathrm{~mm}$ diameter) were considered as type I and those with varicosities in truncal veins ( $>4$ $\mathrm{mm}$ diameter) as type II. Risk ratio was determined by multivariate regression analysis. About $47.67 \%$ of patients in this study were found to have CEAP class 3 disease. Compared with varicose veins of large truncal veins, patients with varicosities in smaller superficial veins had 2.85 -fold $(p<0.01)$ more risk of edema and 5.71-fold $(p<0.01)$ higher prevalence of hyperpigmentation. Varicosities in small superficial veins were associated with higher risk of ulceration (odds ratio 3.93, 95\% confidence interval 2.51-6.18) compared with truncal vein reflux. Their study revealed that presence of small varicose veins in patients without truncal saphenous reflux involvement is associated with severe manifestations of venous insufficiency such as edema and skin lesions even in the absence of varicosities in truncal saphenous veins [18].

Chronic venous insufficiency causes skin pigmentation of the leg ranging from small patches of mild dyschromia to extensive areas of severe skin pigmentation. It is thought that the pigmentation is mainly due to hemosiderin or melanin deposition. A study on 45 patients at Banaras Hindu University categorized the grading of pigmentation into four grades: + , few spots; ++ , pigmentation over gaiter area; +++ , pigmentation involving leg and ankle; ++++ , heavily pigmented (dark). Majority of cases, i.e., 62\% of limbs fall under (++) grade of pigmentation, followed by (+) grade of pigmentation in $20 \%$, while $(+++)$ and $(++++)$ constitute $9 \%$ of the cases each. Increased melanin deposition was seen in 40 pigmented skin biopsies and 3 normal skin biopsies from the case group, and normal melanin deposition was seen in all the non-varicose controls [19, 20]. Ambulatory venous pressure measurement study carried out at Banaras Hindu University correlates with the clinical grading of the CEAP classification of varicose veins. Ambulatory venous pressure measurements do not help in determining the anatomical sites of incompetence. Pressure relief index, the recovery time intervals, and the mean ambulatory venous pressure are the important parameters that can help in identifying and grading the severity of the disease [21].

In a prospective observation study on Indian patients, QOL was approximately $50 \%$ at baseline and improved 
significantly $(\mathrm{P}<0.001)$ after treatment with micronized purified flavonoid fraction (MPFF) [22]. A consensus statement for the management of CVD has been developed and it mainly tell the role of primary doctors in diagnosing this condition along with education to the society [23].

\section{Leg Ulcer}

Compression therapy is the mainstay treatment for venous ulcer [24]. However, in failure cases, surgery can be preferred. Medical management with MPFF as an adjuvant therapy to standard treatment has been reported to be effective and safe in patients with venous ulcer. In addition to standard therapy, diet and lifestyle modification including progressive resistance exercise, patient education, leg elevation, weight reduction, maintaining a healthy cardiac status and strong psychosocial support reduces the risk of recurrence and improves the quality of life in patients with venous ulcer. A consensus was made as an evidence-based management approach for the patients with venous ulcers. Various management options for venous ulcers include compression therapy, minimally invasive procedures like sclerotherapy and ablation techniques, surgical procedures, debridement, and medical management with micronized purified flavonoid fraction (MPFF) $[25,26]$.

In India, many indigenous and traditional home remedies are commonly used including honey, neem oil, and aloe vera. However, there are no evidence based studies to advocate their use in leg ulcers. Honey-impregnated dressings did not significantly improve venous ulcer healing at 12 weeks compared with usual care in a large community-based open-label randomized trial (level B) [27]. Activated charcoal has been tried in malodorous leg ulcers [28]. Boiled potato peel (PP) dressing has been used in chronic leg ulcers (level D) [29]. The PP dressing acts as a barrier, thereby protecting the wound against exogenous agents and secondly the cork layer of the PP prevents dehydration, thereby providing a moist environment which is the single most important factor for wound healing. Honey-impregnated dressings have been used for its antiseptic/antibacterial effects and have shown a favorable outcome in wound healing in burn patients, in comparison to boiled PPs and silver sulfadiazine (level C) [30-32].

\section{Deep Vein Thrombosis}

The prevailing notion that the incidence of VTE in Asians is less than that in the Western population has been disproved by several studies. The incidence of postoperative DVT in Indian patients undergoing major lower limb surgery is as high $(43 \%$ and $60 \%$ patients in the groups with and without prophylaxis, respectively) as seen in the Western world [33-35].

A study was conducted at Pune, Ludhiana, and Bangalore, to provide real-world information on patient characteristics, management strategies, clinical outcomes, and temporal trends in VTE. A multicenter retrospective registry involving 549 medical records of patients with confirmed diagnosis of VTE (deep vein thrombosis [DVT] confirmed by Doppler ultrasonography; pulmonary embolism [PE] by computed tomography, pulmonary angiography and/or V/Q scan) from 2006 to 2010 at three Indian tertiary care hospitals Pune, Ludhiana, and Bangalore. Acute DVT without PE, acute DVT with PE, and PE alone were reported in 64\% (352/549), 23\% (124/549), and $13 \%$ (73/549) patients, respectively. Mean age was $47( \pm 16)$ years, and $70 \%$ were males. H/o DVT (34\%), surgery including orthopedic surgery (28\%), trauma (16\%), and immobilization > 3 days (14\%) were the most common risk factors for VTE. Hypertension (25\%), diabetes (19\%), and neurological disease (other than stroke) (8\%) were the most common co-morbidities. Most $(94 \%)$ were treated with heparin alone $(82 \%)$ or fondaparinux (2\%) for initial anticoagulation; low molecular weight heparin alone $(5 \%)$ or warfarin/acenocoumarol $(76 \%)$ for long-term anticoagulation. Anticoagulant treatment was stopped because of bleeding in 2\% (9/515) patients. Mortality was $7 \%$ among patients diagnosed with VTE during hospital stay versus $1 \%$ in those hospitalized with diagnosed VTE. The annual incidence of DVT ( \pm PE) increased from 2006 to 2010. The study concluded that acute DVT alone was responsible for the substantial burden of VTE in Indian patients. Bleeding was not the limiting factor for anticoagulant treatment in most patients [36].

Several risk factors for deep venous thrombosis (DVT) have been identified, and inherited thrombophilias constitute a significant proportion of them. The most common inherited thrombophilia is activated protein C (APC) resistance, and factor V Leiden is the most common cause of APC resistance. The high prevalence of APC resistance in Caucasians is established, and the prevalence among persons of Asian and African descent has been shown to be low in previous studies. A study conducted at Banaras Hindu University, twenty-three patients with lower limb DVT were included in the study. Diagnosis was confirmed by duplex ultrasonography. Patients were tested for APC resistance with use of the STA Staclot APC-R system (Diagnostica Stago, Asnieres, France). Ten patients (43.5\%) tested positive for APC resistance, while $13(56.5 \%)$ tested negative. APC resistance, although considered a rarity, has been found to have a high prevalence in patients with DVT from the northeastern region of India. APC resistance estimation should be done for all patients with DVT [37].

Pulmonary embolism is one of the commonest cause of sudden unexplained deaths in hospitalized patients [38]. In subset data of ENDORSE, it was found out despite a similar proportion of at-risk hospitalized patients in India and other participating countries, there was major underutilization of prophylaxis in India. It necessitates increasing awareness about VTE risk and ensuring appropriate thromboprophylaxis [39]. 
Post-thrombotic syndrome (PTS) is a late outcome of deep vein thrombosis characterized by cramping pain, swelling, hyperpigmentation, eczema, lipodermato-sclerosis, and ulceration in the leg due to increased venous outflow resistance and reflux venous flow. Newer surgical and endovascular interventions have a promising result in the management of postthrombotic syndrome. Early surgical or endovascular interventions in appropriately selected patients may decrease the incidence of recurrent ulceration and skin changes and provide a better quality of life. Duplex and IVUS (intravenous ultrasound) along with venography serve as cornerstone investigative tools for assessment of reflux and obstruction. Venous obstruction, if present, should be addressed earlier than reflux. It requires endovenous stenting, endophlebectomy, or open bypass procedures. Venous stripping, foam sclerotherapy, radiofrequency, or laser ablation are used to abolish superficial venous reflux. Valvuloplasty procedures are useful for incompetent but intact deep venous valves, while transposition or axillary vein autotransplantation is done for completely destroyed valves [40].

\section{Conclusion}

Venous disease as thought that it is not so common in Indian scenario is not a true statement. More and more studies are needed to look into the epidemiology of various problem especially the chronic venous deficiency and deep venous thrombosis.

\section{Declarations}

Competing interests The authors declare no competing interests.

Open Access This article is licensed under a Creative Commons Attribution 4.0 International License, which permits use, sharing, adaptation, distribution and reproduction in any medium or format, as long as you give appropriate credit to the original author(s) and the source, provide a link to the Creative Commons licence, and indicate if changes were made. The images or other third party material in this article are included in the article's Creative Commons licence, unless indicated otherwise in a credit line to the material. If material is not included in the article's Creative Commons licence and your intended use is not permitted by statutory regulation or exceeds the permitted use, you will need to obtain permission directly from the copyright holder. To view a copy of this licence, visit http://creativecommons.org/licenses/by/4.0/.

\section{References}

1. Khanna AK, Puneet (2010) Manual of vascular surgery. Jaypees Brother Medical publisher, India

2. Khanna AK, Tiwary SK (2016) Ulcers of the lower extremity. Springer, India
3. Khanna AK, Jindal R (2018) Venous disorders. Springer Nature Singapore Pte Ltd.

4. Khanna AK, Tiwary SK (2021) Vascular malformations. Springer Nature Singapore Pte Ltd.

5. Kapadia S (2016) Vascular diseases simplified. Jaypees Brother Medical publisher, India

6. UbramoniamVaidyanathan, Riju Ramachandran Menon, Pradeep Jacob, Binni John (2015) Chronic venous disorders of the lower limb A surgical approach. Springer India

7. Khanna S, Ramkrit R, Tiwary SK, Pathak M, Khanna AK (2021) Anatomy of fascial canal of great sephanous vein. An observational study. Int J Anat Radiol Surg 10(3):A001-A004

8. Hamdan A (2012) Management of varicose veins and venous insufficiency. JAMA 308:2612-2621

9. Bergan JJ, Schmid-Schönbein GW, Smith PD, Nicolaides AN, Boisseau MR, Eklof B (2006) Chronic venous disease. N Engl J Med 355:488-498

10. Malhotra SL (1972) An epidemiological study of varicose veins in Indian railroad workers from the South and North of India, with special reference to the causation and prevention of varicose veins. Int J Epidemiol 1(2):177-183

11. Joseph N, Abhishai B, FaizanThouseef M, Uma Devi M, Abna A, Juneja I (2016) A multicenter review of epidemiology and management of varicose veins for national guidance. Ann Med Surg 8:2127

12. Brand FN, Dannenberg AL, Abbott RD, Kannel WB (1988) The epidemiology of varicose veins: the Framingham Study. Am J PrevMed 4:96-101

13. Mishra S, Ali I, Singh G (2016) A study of epidemiological factors and clinical profile of primary varicose veins. Med J DY Patil Univ 9:617-621

14. Vashist M, Godara R, Sen J, Panwar S (2008) Management of varicose veins: status of clinical examination and colour Doppler in the present Indian scenario. Int J Surg 20:1-4 Available from: http://www.ispub.com/IJS/20/1/7613

15. Katiyar A, Khanna AK, Khanna S, Tiwary S, Nath G (2018) Microbiological study in primary varicose veins. Int Angiol 37(Supplement 1):1-31

16. Tiwary SK, Kumar A, Mishra SP, Kumar P, Khanna AK (2020) Study of association of varicose veins and inflammation by inflammatory markers. Phlebology 35(9):679-685. https://doi.org/10. $1177 / 0268355520932410$

17. Tiwary SK, Alam S, Sureka P, Kumar P, Khanna AK (2020) Study of quality of life in patients with varicose vein after radiofrequency ablation and ultrasound guided foam sclerotherapy. Acta Phlebologica 21(3):42-47

18. Radhakrishnan N, Deepu G, Jayakrishnan R, Sumi S (2018) Vein size and disease severity in chronic venous diseases. Int J Angiol 27(04): 185-189

19. Praveen Kumar KG, Satyendra Tiwary, Ajay Kumar Khanna, Soumya Khanna, Neeraj Dhameeja (2018) Asessment of grading of pigmentation in chronic venous insufficiency. Int Angiol. 37(Supplement 1 to No. 1):53

20. Praveen SKT, Dhameeja N, Puneet Khanna AK, Khanna S (2020) Assessment and grading of pigmentation in chronic venous insufficiency. Phlebology 35(6):394-401

21. Khanna AK, Singh S, Tiwary S, Khanna S, Deshpande S, Shukla R, Kumar P (2020) Ambulatory venous pressure studies and its correlation with CEAP grading of varicose veins. Acta Phlebologica 21(1-2):16-22

22. Pinjala RK, Abraham TK, Chadha SK et al (2004) Long-term treatment of chronic venous insufficiency of the leg with micronized purified flavonoid fraction in the primary care setting of India. Phlebology. 19(4):179-184

23. Dekiwadia D, Jindal R, Varghese R, Bedi HS, Padaria S, Patel M (2016) A consensus statement-part I. Recommendations for the 
management of chronic venous disease (CVD) in India and the key role of primary care doctors. Phlebol Rev 24(1):7-19

24. Tiwary SK, Chaubey K, Khanna S, Kumar P, Khanna AK (2020) Effect of four layer dressing on venous ulcer. Acta Phlebologica 21(3):36-41

25. Prakash S, Tiwary SK, Mishra M, Khanna AK (2013) Venous ulcer: review article. Surg Sci 4:144-150

26. Jindal R, Dekiwadia DB, Krishna PR, Khanna AK, Patel MD, Padaria S, Varghese R (2018) Evidence-based clinical practice points for the management of venous ulcers. Indian J Surg 80(2): 171-182

27. Jull A, Walker N, Parag V, Molan P, Rodgers A (2008) Honey as adjuvant leg ulcer therapy trial collaborators. Randomized clinical trial of honey-impregnated dressings for venous leg ulcers. Br J Surg 95:175-182

28. Sornakumar L, Kalarani M, Srinivas CR (2010) Activated charcoal dressing in malodorous leg ulcers. Indian J Lepr 82:147-148

29. Patange VS, Fernandez RJ, Motla MU, Mahajan SA (1996) Dressing wounds with potato peel. Indian J Dermatol Venereol Leprol 62:286-288

30. Subrahmanyam M (1996) Honey dressing versus boiled potato peel in the treatment of burns: a prospective randomized study. Burns 22:491-493

31. Gupta SS, Singh O, Bhagel PS, Moses S, Shukla S, Mathur RK (2011) Honey dressing versus silver sulfadiazene dressing for wound healing in burn patients: a retrospective study. J CutanAesthetSurg 4:183-187

32. Vishwanath V (2014) Venous leg ulcer: management aspect in Indian scenario. Indian Dermatol Online J 5:396
33. Lee LH, Gu KQ, Heng D (2002) Deep vein thrombosis is not rare in Asia - the Singapore General Hospital experience. Ann Acad Med Singap 31:761-764

34. Dhillon KS, Askander A, Doraismay S (1996) Postoperative deepvein thrombosis in Asian patients is not a rarity: a prospective study of 88 patients with no prophylaxis. J Bone Joint Surg (Br) 78:427430

35. Agarwala S, Bhagwat AS, Modhe J (2003) Deep vein thrombosis in Indian patients undergoing major lower limb surgery. Indian J Surg 65:159-162

36. Kamerkar DR, Joseph John M, Desai SC, Dsilva LC, Joglekar SJ (2016) Arrive: a retrospective registry of Indian patients with venous thromboembolism. Indian J Crit Care Med 20(3):150-158

37. Agrawal N, Kumar S, Puneet RK, Shukla J, Khanna AK (2009) Activated protein $\mathrm{C}$ resistance in deep venous thrombosis. Ann Vasc Surg 23:364-366

38. Kapoor VK (2010) Venous thromboembolism in India. Natl Med J India 23:193-195

39. Ramakrishna Pinjala on behalf of all ENDORSE India investigators (2012) Venous thromboembolism risk \& prophylaxis in the acute hospital care setting (ENDORSE), a multinational cross-sectional study: results from the Indian subset data. Indian J Med Res 136: 60-67

40. Khanna AK, Shivanshu S (2012) Post thrombotic syndrome - surgical possibilities. Thrombosis 2012:520604

Publisher's Note Springer Nature remains neutral with regard to jurisdictional claims in published maps and institutional affiliations. 